\title{
Robust Capacity Control in Revenue Management: A Literature Review
}

\author{
Zheyu Jiang \\ School of Business Administration, South China University of Technology, Guangzhou, China \\ Email: zheyjiang@126.com
}

How to cite this paper: Jiang, Z.Y. (2018) Robust Capacity Control in Revenue Management: A Literature Review. Open Journal of Business and Management, 6, 488-497.

https://doi.org/10.4236/ojbm.2018.62037

Received: April 3, 2018

Accepted: April 27, 2018

Published: April 30, 2018

Copyright $\odot 2018$ by author and Scientific Research Publishing Inc. This work is licensed under the Creative Commons Attribution International License (CC BY 4.0).

http://creativecommons.org/licenses/by/4.0/ (c) (i) Open Access

\begin{abstract}
Revenue management is one of the classical topics in operations research. In recent 10 years, robust optimization methodology motivated a rapid growing amount of literature on robust revenue management. This stream of research does not rely on strict prior assumptions on distribution of random demand in classical revenue management theory but is capable to maintain tractable and to derive interesting structural properties of optimal solution. In this paper, we first briefly introduce theoretical basis of revenue management and then provide detailed review of literature on robust revenue management. Then potential directions of future research are identified.
\end{abstract}

\section{Keywords}

Revenue Management, Robust Optimization, Capacity Control, Pricing Control

\section{Introduction}

Revenue management is one classical research topic in operation research and management science field. In classical textbook such as [1], it is succinctly defined as "selling the right product to the right customers at the right time". It entails capacity control, pricing control and other control methodology for managers or firms to increase sales and revenue with inflexible capacity. Research in this filed emerges in 1970s when business practice of the US airline industry changed and airlines were free to change prices, schedules and service without public regulation. After being investigated for over 40 years, revenue management has become a fundamental component of management science theory, and has been successfully applied in many other industries, such as airline cargo service firms, retail chains, hotel companies, car rental companies, in- 
ternet service providers and electric power suppliers etc.

One of significant forces which facilitate the development of revenue management is scientific advances in economics, statistics and operation research [2]. Advances in these fields are helpful to model demand, economic conditions, qualify the uncertainties faced by firms or managers. Nowadays, there exists a new trend in revenue management which is driven by one new optimization methodology, called robust optimization.

In the following part, we first briefly introduce basic hypothesis, representative research topics and control methods in classical revenue management.

\subsection{Revenue Management}

In revenue management, managers face a fundamental trade-off between current revenue with higher potential revenue in the future. On the one hand, the firms would like to sell their products or capacity to customers who have higher evaluation to earn higher revenue. On the other hand, the firms are faced with risks of waiting too much time for valuable customers but failed to sell products in limited time. Common topics in revenue management include single-resource capacity control, network capacity control, overbooking, dynamic pricing and auctions.

There are three common assumptions in classical revenue management (see [2]). First, demands faced by the firms are uncertain and usually characterized as random variables. In most cases, demands in revenue management show multi-dimensional nature including different types of products and customers or long periods. Secondly, most revenue management is based on the concept of the "rationality", i.e. the firms' objective is to maximizing profit or minimizing cost. Finally, the firms usually face production inflexibility. According to [2], joint production constraints and costs complicate the revenue management. The firms can diminish variations in demand by utilizing variations in supply. With inflexible supply, it is difficult for firms to match random demand with supply.

In the recent 10 years, one new optimization methodology which can make optimal solutions considering parameter uncertainty arises up. In contrast to stochastic optimization which starts by assuming a probabilistic description of the uncertainty, robust optimization models uncertainty as deterministic and set-based. Robust optimization has been investigated in many traditional optimization-theoretic concepts such as algorithms, tractability as well as modeling power and structural analysis. It also affects revenue management theory profoundly.

\subsection{New Trend}

Even stochastic programming has been studied over several decades, it still has some limits in theory and practice. On the one hand, stochastic requires exact prior knowledge of random variables however it is difficult for decision makers to collect enough data to derive exact estimation. On the other hand, according 
to structure properties of solutions to classical stochastic program, even a slight error in estimating parameters could lead to large deviation of optimal solution to the original problem [3]. Thus researchers are seeking for more robust solutions which can resist ambiguity in data collection and estimation.

In terms of methods to describe uncertainty in parameters, there are two kinds of robust optimization methods. One type of the uncertainty sets are deterministic with common topology structure such as ellipsoidal uncertainty set and polyhedral uncertainty set. [4] first studies on the truss topology design problem with respect to given loading scenarios and small "occasional "loads. They show that resulting program is a semi-definite program. Several equivalent forms of the program are also presented. [5] formulates robust linear program with ellipsoidal uncertainty set. They build up one convex robust counterpart of the uncertain linear program to develop analytical and computational optimization tools. They proved that the robust counterpart of the linear program is a conic quadratic program, which can be solved efficiently. [6] defines one robust linear program with polyhedral uncertainty sets. By relaxing and taking the dual of the inner maximization problem in constraints, they show a equivalent linear formulation which is also tractable.

Another type of literature assumes that decision makers do not know the exact distribution but know parameters (such as support, mean or covariance matrix etc.) of the distribution. [7] proves that for a wide range of cost functions, the distributionally robust stochastic program can be solved efficiently. [8] applies the distributionally robust optimization to study the newsvendor problem with limited demand information and obtain closed-form or tractable solutions. Because of the large amount of literature in robust optimization and our goal to study robust capacity control in revenue management, we do not list all literature in basic optimization methodology. Interested readers can refer to related literature review, such as [3] and [9].

\subsection{Outline}

The remainder of this paper is structured as follows: In Section 2, we briefly introduce classical revenue management. We explain the existing research works in revenue management in two main categories: capacity control and pricing control. In Section 3, we review most important literature on robust revenue management. We also classify all related literature into two parts by the type of control method. In the Section 4, we identify a number of potential directions for future research.

\section{Classical Revenue Management}

[2] classifies classical revenue management research according to control method. The first type is to manage revenue by capacity control (quantity-based revenue management). Researchers seek to design optimal method to allocate limited capacity to different classes or bundles of demand at given prices. Two 
successful examples are managing the sales of different fare classes on flights or controlling the sales of different rooms in hotels. The other type is to control sales by setting right prices (price-based revenue management). Research in this field is based classical assumption in economy which demand of one product is affected by its price. By dynamically setting prices, the firms can control the demand and sales of their products. In this section, we aim to introduce basic research questions and control methods in revenue management, thus we just review some fundamental papers in revenue management.

\subsection{Capacity Control}

In this part, we introduce the concept of capacity control in revenue management. Managers apply robust control in revenue management by allocating capacity of one or multiple resources to different classes of demand. In most cases, demands from different customers are classified by prices that they offer. For example, in single-leg flight capacity control problem, airline allocates all seats to different classes of customers who are classified by prices they offer. In classical revenue management theory, there are three basic capacity-based controls to allocate capacity: booking limits, protection levels and bid prices. Booking limits are controls that limit the amount of capacity that can be sold to any particular class at a given point in time. To increase the utilization of capacity, firms prefer to set nested booking limit. Protection levels specify an amount of capacity to reserve for a particular class or set of classes. Similarly, firms prefer to set nested protection levels. Booking limit and protection levels are alternative controls. Bid prices are one control method that firms set a threshold price such that a request is accepted if its revenue exceeds the threshold price and rejected otherwise. The textbook [2] offers detailed overview and references about capacity-based revenue management.

\subsection{Pricing Control}

Price control is a more natural mechanism in practice. Managers apply it by setting appropriate prices to control demand and optimize revenue. Pricing control includes different forms such as personalized pricing, markdowns, display and trade promotions, coupons and discounts, clearance sales, auctions and price negotiations. To optimize policies with uncertain demand, more research developed stochastic models to study on price-based revenue management. Single-product dynamic pricing is one basic research problem in revenue management which establishes theoretical basis for more complex problems. [10] first studies dynamic pricing problem of a single product over a finite sales horizon given a fixed inventory at the start of the sales horizon. They formulate the problem using intensity control and obtain structural monotonicity results for the optimal intensity as a function of the stock level and the length of the horizon. They also find optimal pricing strategy in closed form for one particular exponential family of demand functions and prove that simple fixed price poli- 
cies are asymptotically optimal as the expected sales tends to infinity. For two-price model where prices are fixed in each period and firms can only decide to switch from one to the other, [11] obtain structural properties of the optimal stopping time problem. They prove that the optimal policy is of a threshold type.

There are some other detailed textbooks or literature on classical dynamic pricing problem, see [1] [2]. For more recent research, [12] provides an in-depth overview of the available literature on dynamic pricing and learning. [13] identifies most recent trends in dynamic pricing research involving pricing with multiple products, pricing with competition and pricing with limited information.

\section{Robust Revenue Management}

During the last 10 years, researchers have been developing robust revenue management policy. In addition to the scientific advances in the optimization methods, there are other motivations for researcher to focus on this problem.

In this part, we will first explain some advantages of robust revenue management comparing to classical revenue management. First, robust revenue management does not require much prior knowledge. Robust capacity control or price control can be employed with limited or without prior data about uncertainty. This allows robust control to be applied in situations which is more difficult to collect data. For example, in ocean freight industry, firms often set prices by unofficial and infrequent negotiation (for example, see [14]). Secondly, robust revenue management shows computational tractability and efficiency. For example, [15] develops polynomial time algorithm to solve robust dynamic pricing problem.

\subsection{Robust Capacity Control}

[16] proves that when the seller knows only the upper and lower bounds of the demand, the network revenue management problem (multiple products use multiple resources) using maximin and the minimax regret criteria are both NP-hard. Then they discuss several special but tractable revenue network: single resource revenue management, bundle revenue management. Finally, a heuristic algorithm is given for general cases. [17] respectively discusses robust version of static and dynamic single resource capacity allocation, and designs polynomial time algorithm. [18] introduces competitive ratio criteria to robust revenue management from the perspective of online algorithm in computing science. For two fare classes, multiple fare classes and bid-price controls problem, they obtain the lower bounds of competitive ration and corresponding protection level or biding price. Notice that [18] assume that seller knows no information about uncertain demand.

[19] employs competitive analysis to study single resource multi-fare revenue management problem. [19] assumes that the seller knows only the upper and lower bounds of the demand of each product. The competition ratio and absolute regret value are taken as performance criteria. They prove that the optimal 
capacity control policies under both criteria are nested booking limit. [20] studies the robust version of another classical problem in revenue management-overbooking. They develop a model of overbooking and fare-class allocation in multi-fare, single-resource problem. They obtain optimal overbooking levels and booking limits in closed form which are nested. [21], in order to avoid the spiral drop effect produced by "truncated" demand, uses entropy to estimate the total demand and introduce the capacity control revenue management problem of the two products. Finally, the convergence of the algorithm is proved.

\subsection{Robust Pricing Control}

[22] establishes a robust optimization model for single product dynamic pricing problem, where uncertain demand belongs to a given polyhedron uncertainty set. Then, [22] obtains the tractable convex optimization form which is equivalent to the original model. The new form has only one new variable called reference price variable. The article shows that when the uncertainty of demand increases, the optimal price is gradually converging to the reference price. They also show that if the price is low and the uncertainty is strong, the price below the nominal value is not the most important decision. [23] uses the same modeling method to model the multi-product pricing problem, and shows that when the product uses the same resource, the equivalent pricing form is inverse convex programming. When a product uses multiple resources at the same time, the equivalent form is non-convex programming. Next, [23] proposed the lower bound for second problem.

[24] studies monopoly pricing problem under incomplete information. The seller only knows that the real demand belongs to one given uncertainty set. They compare two models with two different objectives, maximizing minimal utility and minimizing maximal regret value. They prove that the equilibrium price under two goals is lower than the price when demand is fixed. In order to ensure the robustness of decision-making, the seller needs to pay more information rents (information rent). [25] studies how the seller set price when the seller knows the least information about the demand, that is, when the support of the consumer value is only known. The objective of the model is to minimize the maximal regret value. The article shows that consumers with lower value will be excluded from the market at high prices.

[26] study the dynamic pricing problem of single product. Unlike the previous ones, the paper studies Markovian pricing strategy, and uses the notion of relative entropy to represent the uncertainty in demand rate model. In this paper, they use the probability measure knowledge of point process to transform the original model and get the solvable reformulation. Finally, Isaacs equation for stochastic differential games is applied to obtain optimal pricing policy. In particular, they give the closed-form solutions for the exponential nominal demand rate model. [27] extends the modeling method of [26] to multi product case. The paper considers that the demand for different products have different levels of 
uncertainty and proves that this problem is equivalent to a risk sensitive dynamic pricing problem. Under some additional assumptions, they show some features of the model and characterize the revenue sharing rule which leads to equivalence between the risk-sensitive pricing problem.

[15] proposes a polynomial time algorithm for the two stage dynamic pricing model under minimizing the maximal regret value. [28] studies the problem of robust pricing under limited information or different learning ability, and establishes a model with the objective of minimizing the competition ratio (competitive ratio). For each situation, the optimal solution in the form of closed-form is given. [29] studied the dynamic pricing problem of strategic consumers, and designed approximate algorithm to solve it. Numerical results show that this kind of control has greatly improved the average revenue compared with previous studies. Unlike above research, [30] studies the dynamic pricing problem of multiple products with oligopoly. Such a model is a parametric pricing strategy, that is, the parameter of the demand function is uncertain which belongs to a given polyhedron set. It is proved that the solution of the model is equal to the solution of the system of quasi-variational inequalities, and it is proved that there exists an equilibrium solution for the inequality group. Finally, the iterative learning algorithm is designed to find out the equilibrium solution.

\section{Future Research Directions}

We have provided a review of research in robust revenue management. In this section, we identify some potential directions for future research in robust revenue management.

First, there is still a gap between classical revenue management theory and robust revenue management. For example, there is few literature on robust auction mechanism design. Comparing to dynamic pricing problem, in the auction problem, one customer offer a price to bid one product and then firms decides which bids to accept. Auction problems are common in practice. This mechanism has been applied in many industries such as used cars, art, real state and electricity etc. In classical auction theories, equilibrium strategies are based on some prior assumptions of uncertain factors, for example, the distribution of customer's private evaluation. These assumptions may not be satisfied in reality, thus study on robust auction mechanism is of great importance.

Second, data-driven research provides new methodology for classical revenue management. With the rapid growth of IT, researcher are facing a new era when enormous amount of data are generated and stored. "Big data" era presents new opportunities as well as challenges for researchers in different fields including revenue management. For example, [31] study a dynamic pricing problem to determine a robust and dynamic pricing strategy. Comparing to existing work, they develop a tractable optimization model which can directly use demand sample data. In addition to their work, it is interesting to see how capacity control and pricing control can be extended in robust data-driven setting. 


\section{Summary}

In this work, we reviewed the literature on robust revenue management. We first briefly introduce the definition, common research problems and assumptions in revenue management. Then, to present the contribution of robust revenue management, we introduce basic concept of classical revenue management. Subsequently, we conducted a bibliographic survey of the literature on robust revenue management. Finally, we identify some potential directions for research in the future.

Robust revenue management applies robust optimization to revenue management topics. The literature has investigated robust capacity control and robust pricing control in revenue management. Some results of the research works have been demonstrated, such as nested booking limits, closed-form solution and efficient computation. On the other hand, some topics are still interesting to be investigated such as robust auction problem and data-driven revenue management.

\section{Acknowledgements}

Author thanks the reviews and the editor.

\section{References}

[1] Bitran, G. and Caldentey, R. (2003) An Overview of Pricing Models for Revenue Management. Manufacturing \& Service Operation Management, 5, 203-229. https://doi.org/10.1287/msom.5.3.203.16031

[2] Talluri, K.T. and Van Ryzin, G.J. (2005) The Theory and Practice of Revenue Management. Springer, New York.

[3] Ben-Tal, El Ghaoui, L.and Nemirovski, A. (2009) Robustness Optimization. Princeton University Press, Princeton.

[4] Ben-Tal and Nemirovski, A. (1997) Robust Truss Topology Design via Semidefinite Programming. SIAM Journal of Opimization, 7, 991-1016.

https://doi.org/10.1137/S1052623495291951

[5] Ben-Tal and Nemirovski, A. (1998) Robust Solutions of Uncertain Linear Programs. Operations Research Letter, 23, 769-805.

[6] Bertsimas, Dimitris and Sim, M. (2011) The Price of Robustness. Operations Research, 52, 35-53. https://doi.org/10.1287/opre.1030.0065

[7] Delage, E and Ye, Y. (2010) Distributionally Robust Optimization under Moment Uncertainty with Application to Data-Driven Problems. Operations Research, 58, 595-612. https://doi.org/10.1287/opre.1090.0741

[8] Perakis, G. and Roels, G. (2008) Regret in the Newsvendor Model with Partial Information. Operations Research, 56, 188-203.

https://doi.org/10.1287/opre.1070.0486

[9] Bertsimas, Dimitris, Brown, D.B. and Caramanis, C. (2011) Theory and Applications of Robust Optimization. SIAM Review, 53, 464-501.

https://doi.org/10.1137/080734510

[10] Gallego, G. and van Ryzin, G. (1994) Optimal Dynamic Pricing of Inventory with Stochastic Demand over Finite Horizons. Management Science, 40, 999-1020. 
https://doi.org/10.1287/mnsc.40.8.999

[11] Feng, Y. and Gallego, G. (1995) Optimal Starting Times for End-of-Season Sales and Optimal Stopping Times for Promotianl Fares. Management Science, 41, 1371-1391. https://doi.org/10.1287/mnsc.41.8.1371

[12] den Boer, A. (2015) Dynamic Pricing and Learning. Surveys in Operations Research and Management Science, 20, 1-18. https://doi.org/10.1016/j.sorms.2015.03.001

[13] Chen, M. and Chen, Z.-L. (2015) Recent Developments in Dynamic Pricing Research: Multiple Products, Competition, and Limited Demand Information. Production and Operations Management, 24, 704-731. https://doi.org/10.1111/poms.12295

[14] Lee, C.Y., Tang, C.S., Yin, R. and An, J. (2015) Fractional Price Matching Policies Arising from the Ocean Freight Service Industry. Production and Operations Management, 24, 1118-1134. https://doi.org/10.1111/poms.12337

[15] Chen, Z.L., Hall, N.G. and Keller, H. (2017) Dynamic Pricing to Minimize Maximum Regret. Production and Operations. Management, 26, 47-63. https://doi.org/10.1111/poms.12608

[16] Perakis, G. and Roels, G. (2010) Robust Controls for Network Revenue Management. Manufacturing \& Service Operations Management, 12, 56-76. https://doi.org/10.1287/msom.1080.0252

[17] Birbil, S.I., Frenk, J.B.G., Gromicho, J.A.S. and Zhang, S. (2009) The Role of Robust Optimization in Single-Leg Airline Revenue Management. Management Science, 55, 148-163. https://doi.org/10.1287/mnsc.1070.0843

[18] Ball, M. and Queyranne, M. (2009) Towards Robust Revenue Management Competitive Analysis of Online Booking. Operations Research, 57, 950-963. https://doi.org/10.1287/opre.1080.0654

[19] Lan, Y., Gao, H., Ball, M. and Karaesmen, I. (2008) Revenue Management with Limited Demand Information. Management Science, 54, 1594-1609.

https://doi.org/10.1287/mnsc.1080.0859

[20] Lan, Y., Ball, M. and Karaesmen, I. (2011) Regret in Overbooking and Fare-Class Allocation for Single Leg. Manufacturing \& Service Operations Management, 13, 194-208. https://doi.org/10.1287/msom.1100.0316

[21] Maglaras, C. and Eren, S. (2015) A Maximum Entropy Joint Demand Estimation and Capacity Control Policy. Production and Operations Management, 24, 438-450. https://doi.org/10.1111/poms.12243

[22] Thiele, A. (2006) Single-Product Pricing via Robust Optimization. Working Paper, Department of Industrial and Systems Engineering, Lehigh University, Bethlehem.

[23] Thiele, A. (2009) Multi-Product Pricing via Robust Optimization. Journal of Revenue and Pricing Management, 8, 67-80. https://doi.org/10.1057/rpm.2008.41

[24] Bergemann, D. and Schlag, K. (2011) Robust Monopoly Pricing. Journal of Economic Theory, 146, 2527-2543. https://doi.org/10.1016/j.jet.2011.10.018

[25] Bergemann, D. and Schlag, K. (2008) Pricing without Priors. Journal of the European Economic Association, 6, 560-569. https://doi.org/10.1162/JEEA.2008.6.2-3.560

[26] Lim, A.E.B. and Shanthikumar, J.G. (2007) Relative Entropy, Exponential Utility, and Robust Dynamic Pricing. Operations Research, 55, 198-214. https://doi.org/10.1287/opre.1070.0385

[27] Lim, A.E.B., Shanthikumar, J.G. and Watewai, T. (2010) Robust Multi-Product 
Pricing. Working Paper, Department of Industrial Engineering and Operations Research, University of California, Berkeley.

[28] Eren, S.S. and Maglaras, C. (2010) Monopoly Pricing with Limited Demand Information. Journal of Revenue and Pricing Management, 9, 23-48. https://doi.org/10.1057/rpm.2009.41

[29] Chen, Y.W. and Farias, V.F. (2016) Robust Dynamic Pricing with Strategic Customers. Working Paper, Sloan School of Management, MIT, Cambridge.

[30] Perakis, G. and Sood, A. (2006) Competitive Multi-Period Pricing for Perishable Products: A Robust Optimization Approach. Mathematical Programing, 107, 295-335. https://doi.org/10.1007/s10107-005-0688-y

[31] Cohen, M.C., Lobel, R. and Perakis, G. (2018) Dynamic Pricing through Data Sampling. Production and Operations Management. 